NEUROGASTROENTEROLOGY

\title{
Severe idiopathic gastroparesis due to neuronal and interstitial cells of Cajal degeneration: pathological findings and management
}

\author{
N Zárate, F Mearin, X-Y Wang, B Hewlett, J D Huizinga, J-R Malagelada
}

Gut 2003;52:966-970

Delayed gastric emptying can be due to muscular, neural, or humoral abnormalities. In the absence of an identified cause, gastroparesis is labelled as idiopathic. We present the case of a patient with severe idiopathic gastroparesis. Pharmacological approaches failed, as well as reduction in gastric emptying resistance with pyloric injection of botulinum toxin and pyloroplasty. Therefore, subtotal gastrectomy was performed. Histological and immunohistochemical study of the resected specimen showed hypoganglionosis, neuronal dysplasia, and a marked reduction in both myenteric and intramuscular interstitial cells of Cajal. To our knowledge, this is the first time these rare histological findings have been described in a patient with idiopathic gastroparesis.

U nderstanding of both normal and abnormal gastrointestinal function has gained momentum in recent decades. However, gastric motility and, in particular, the relative contribution of all of the factors involved in the coordination of food propulsion are still poorly understood. Gastric emptying reflects the integration of tonic contractions of the proximal stomach (fundic tone), phasic contractions of the antrum, and the inhibitory forces of pyloric and duodenal contractions. These complex phenomena require cooperation between smooth muscle, enteric and autonomic nerves, and interstitial cells of Cajal (ICC).'

Gastroparesis is a syndrome caused by ineffective propulsion of intragastric contents, indistinguishable clinically from true mechanical obstruction. ${ }^{2}$ Severity of symptoms varies enormously. In mild cases, patients present with early satiety, nausea, and mild abdominal pain, symptoms which, albeit annoying, are not life threatening. On other occasions, symptoms are highly incapacitating and patients suffer abdominal pain and chronic vomiting which may lead to dehydration and nutritional impairment. In almost 50\% of cases no apparent cause is identified ${ }^{2}$; thus the diagnosis of idiopathic gastroparesis (IG) is established.

Several motor disorders of the gastrointestinal tract show disruption of ICC networks in addition to abnormal innervation: Hirschsprung's disease, ${ }^{3}$ hypertrophic pyloric stenosis, and pseudo-obstruction. ${ }^{5}$ ICC are responsible for the generation of the electrical slow wave activity that determines frequency and propagation of peristaltic contractions, ${ }^{6}$ and serve as mediators of inputs from the enteric nervous system in gastrointestinal smooth muscle cells. ${ }^{7}$ Remarkably, data regarding IG pathology and, particularly, ICC distribution are scarce in the medical literature.

We describe the case of a young woman who presented with severe IG with intractable nausea, chronic vomiting, abdominal pain, and marked weight loss. An intense and prolonged medical approach failed to relieve her symptoms, leading ulti- mately to subtotal gastrectomy. Immunohistochemistry revealed degeneration of the myenteric plexus combined with loss of ICC, findings not previously described in the medical literature. IG diagnosis and therapeutic management are addressed. Histological findings and their implications are also discussed.

\section{CASE REPORT}

A 32 year old woman was referred to the Hospital General Universitari Vall d'Hebrón because of intractable gastroparesis. She presented seronegative sacroileitis. She complained of vomiting, bloating, early satiety, and abdominal pain starting 10 years earlier. Physical and neurological examinations revealed no abnormalities, and no signs of autonomic insufficiency were observed. She was slim but not cachectic. A full chemistry and hormonal profile proved normal, including renal and liver function, ions, glucose, fibrinogen, erythrocyte sedimentation rate, thyroid hormones, and antinuclear antibody titres. Prior to admission, upper gastrointestinal series and endoscopy showed a dilated atonic stomach without outlet obstruction or macroscopic lesions. A biopsy specimen of the jejunum showed normal villous height and a mild increase in inflammatory cells in the lamina propria. Whipple's disease was also ruled out. Her bowel movements were normal as were the aspect of the stools. Colonoscopy failed to show any macroscopic abnormality and rectal mucosal biopsy appeared normal. Serum antibodies of the Herpes class viruses were negative and no evidence of mycoplasma infection was found.

Scintigraphy showed severe delayed emptying for both liquids and solids (38\% gastric retention of solid meal at four hours). Brain magnetic resonance performed to rule out a central origin of her symptoms showed no abnormalities.

She was treated unsuccessfully with cholinergic agents and metoclopramide. Then, intravenous infusion of erythromycin at a dose of $250 \mathrm{mg} / 8 \mathrm{~h}$ was started and symptoms improved remarkably. She was discharged initially on oral erythromycin and, afterwards, on cisapride.

Two years after the first admission, she was again referred to our centre. At that time, she was taking both metoclopramide and cisapride but was unable to tolerate even liquids. On this occasion erythromycin failed to relieve her symptoms. Vomiting and abdominal pain continued even with total parenteral nutrition. Thus intrapyloric injection of botulinum toxin (BT) was performed to decrease pyloric resistance and improve gastric emptying. However, this approach provided neither clinical relief nor an improvement in isotopic gastric emptying. Lack of response to pharmacological therapy and

Abbreviations: ICC, interstitial cells of Cajal; IG, idiopathic gastroparesis; BT, botulinum toxin; PGP, protein gene product. 
severity of symptoms prompted us to adopt a surgical approach. Pyloroplasty without selective vagotomy was first attempted. Again, scintigraphy showed no significant change compared with previous observations; however, as symptoms appeared to improve, the patient was discharged. Unfortunately, a few months later she was again referred to our hospital because of food intolerance and exacerbation of her symptoms. At that time we decided to perform total gastrectomy. Before this, extension of gastric motor abnormality, confirmation of intestinal motility indemnity, and tolerance to a nutrient load was sought.

Total gastrectomy with a gastroenteric anastomosis of the "Roux-en-Y" type was then performed. Symptoms improved greatly; she was able to tolerate solid food and gained $12 \mathrm{~kg}$ in one year.

\section{MATERIALS AND METHODS}

\section{Gastrointestinal motor function evaluation}

We used the gastric barostat to evaluate fundic compliance. Standardised gastric distensions were performed by means of an electronic barostat, ${ }^{8}$ using a previously published method.' Pressure and volume inside the intragastric bag were continuously recorded on a paper polygraph (model 1600; MFE, Salem, New Hampshire, USA). The patient was intubated after a 12 hour fast and aspiration of gastric contents. The bag of the barostat, finely folded, was introduced through the mouth into the stomach. To unfold the intragastric bag, one lumen of the connecting tube was connected to a pressure transducer and the bag slowly inflated through the other lumen of the tube with $300 \mathrm{ml}$ of air under controlled pressure $(<20 \mathrm{~mm}$ $\mathrm{Hg}$ ). The bag was then completely deflated and connected to the barostat. After a 15 minute equilibration period, minimal intragastric distending pressure was determined. During the study, we set a baseline intragastric pressure level of $2 \mathrm{~mm} \mathrm{Hg}$ more than the minimal distending pressure. Using the pressure selection dial of the barostat, intermittent phasic distentions were applied at regular intervals ( 10 minutes) in stepwise increments $(4 \mathrm{~mm} \mathrm{Hg})$. Graded distension was induced up to the pressure level that first provided an intrabag volume $>900 \mathrm{ml}$. Results were compared with those previously obtained from 12 healthy volunteers. To measure gastric compliance, we averaged intrabag volumes during each pressure step, and volumes at each pressure level were corrected for air compressibility using Boyle's law $\left(\mathrm{P}_{1} \mathrm{~V}_{1}=\mathrm{P}_{2} \mathrm{~V}_{2}\right.$ ). A compliance curve (volume $v$ pressure) was then constructed. After a 15 minute equilibration period, basal gastric tone at minimal distention pressure $+2 \mathrm{~mm} \mathrm{Hg}$ was also measured for another 15 minutes.

We used gastrointestinal manometry to evaluate extension of the motor defect. The procedure has previously been described in detail. ${ }^{10}$ In brief, an 8 lumen orointestinal tube $(5$ $\mathrm{mm}$ outer diameter) was placed under fluoroscopic guidance with six recording sites $(1 \mathrm{~cm}$ apart) laying across the gastroduodenal junction, with two aboral recording sites (10 $\mathrm{cm}$ apart) in the descending and distal duodenum, respectively. The probe was connected to a low compliance manometric perfusion system. ${ }^{11}$ Antrointestinal pressure activity was recorded continuously on an eight channel paper polygraph (Dynograph Recorder R611; SensorMedics, Anaheim, California, USA) for three hours during fasting and for two hours after ingestion of a $435 \mathrm{kcal}$ solid-liquid meal.

Small bowel tolerance to a nutrient load was tested by placing a nasojejunal tube and infusing Intralipid via a peristaltic pump. Infusion was started at $0.5 \mathrm{kcal} / \mathrm{min}$ and gradually increased to $1.5 \mathrm{kcal} / \mathrm{min}$.

\section{Histological examination}

The resected stomach seemed uniformly dilated and the mucosal surface appeared normal. Sections were stained with haematoxylin-eosin and additional sections were stained with

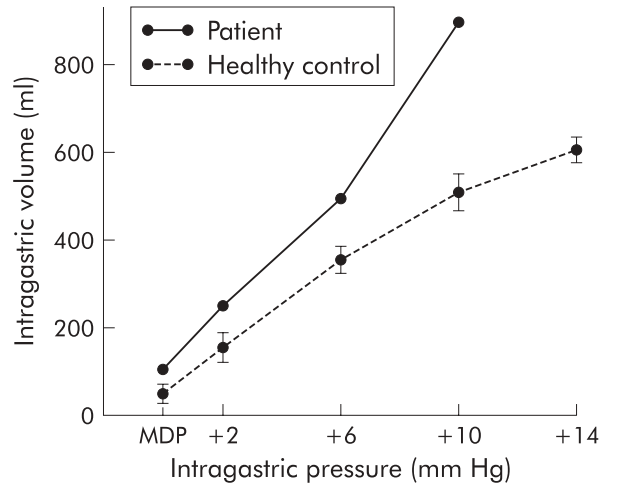

Figure 1 Gastric compliance (volume-pressure relationship) was significantly increased in the patient with idiopathic gastroparesis compared with healthy control values.

Gomori's trichrome. Immunohistochemistry was performed with rabbit anti-kit (Santa Cruz Biotechnology, California, USA) as a marker for ICC, and rabbit anti-protein gene product (PGP 9.5; Ultraclone Ltd, UK) as a marker for nerves. Slides were counterstained with Mayer's haematoxylin and images obtained with a digital camera (Sony 3CCD, Model No DXC-930, Japan). Control experiments involved incubation of sections with phosphate buffered saline from which primary antibodies were omitted. Control gastric tissue was obtained from one individual who had a fatal car accident and no known gut disease. Histological examination of the fundus, antrum, and pylorus was performed in both patient tissue and control tissue.

We quantified c-kit and PGP 9.5 immunostaining. Squares were selected such that the area was maximally covered by the whole musculature $\left(0.5 \mathrm{~mm}^{2}\right)$, including Auerbach's plexus. Six areas were selected from control corpus and antrum and similar regions from the patient. Quantification of kit and PGP 9.5 positivities were performed on a Carl Zeiss Vision Imagine Analysis System workstation using the KS400 program. Kit and PGP 9.5 immunopositive cells were identified and highlighted, maximising ICC detection while minimising background staining. Regions with increased background staining, occasionally found at the borders of the image, were excluded from the analysis. The area of immunopositive cells was calculated and expressed as a percentage of the total area of the image.

Values are reported as mean (SEM). Statistical comparisons were performed using the $t$ test, and a p value of $<0.05$ was taken as statistically significant.

\section{RESULTS}

\section{Gastrointestinal motor function evaluation}

Gastric compliance was significantly greater in the patient than in healthy volunteers (fig 1). The barostat study also showed a low basal gastric tone: mean intrabag volume measured by the barostat over 15 minutes at a constant minimal distention pressure $+2 \mathrm{~mm} \mathrm{Hg}$ was 356 (16.4) ml.

Gastrointestinal manometry provided antral hypomotility (antral motility index 5.4: postcibal antral hypomotility by our laboratory criteria-that is, values less than the mean \pm 2 SD (13.1 (0.2)) with normal intestinal motility.

Nasojejunal infusion of $1.5 \mathrm{kcal} / \mathrm{min}$ over 24 hours was well tolerated by the patient and neither abdominal symptoms nor diarrhoea was observed.

\section{Histological examination}

The mucosal, submucosal, and smooth muscle architecture appeared normal. No Helicobacter pylori forms were found. No intranuclear or viral inclusions were observed. Acute inflammatory cells were absent but CD3 and CD8 immunostaining 


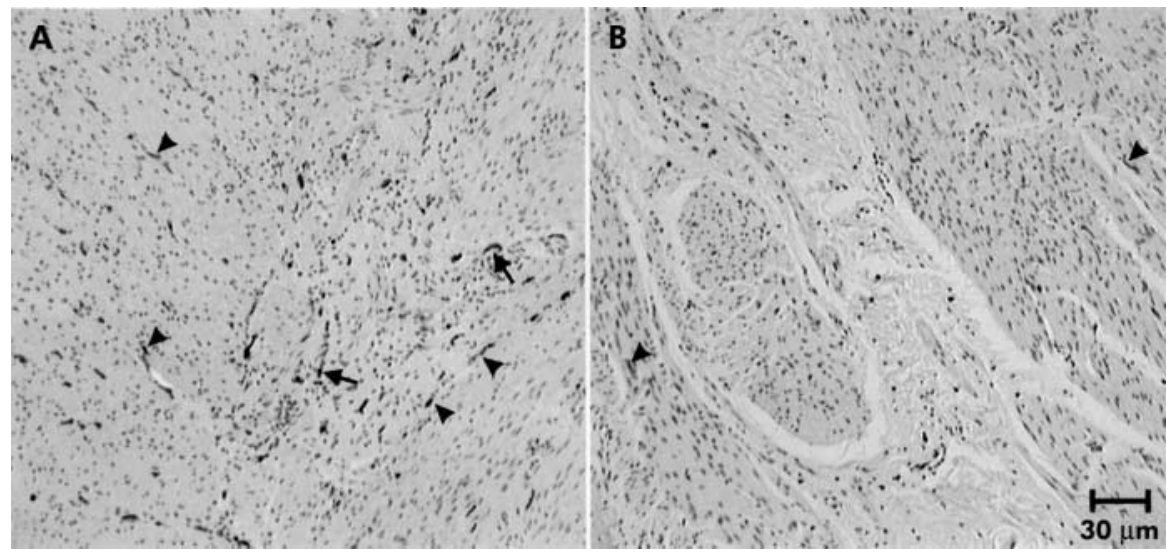

Figure 2 Kit immunoreactivity (IR) in the normal corpus (A) and corpus with gastroparesis (B). (A) Kit-IR is distributed throughout the muscle layers. Some kit-IR cells are concentrated at the myenteric plexus level (arrows). A fair amount of intramuscular kit-IR cells are seen in both muscle layers (arrowheads) running parallel to the muscle cells in which they are located. In (B), however, less kit-IR is found in the whole muscle layers except for a few scattered kit-IR cells (arrowheads).
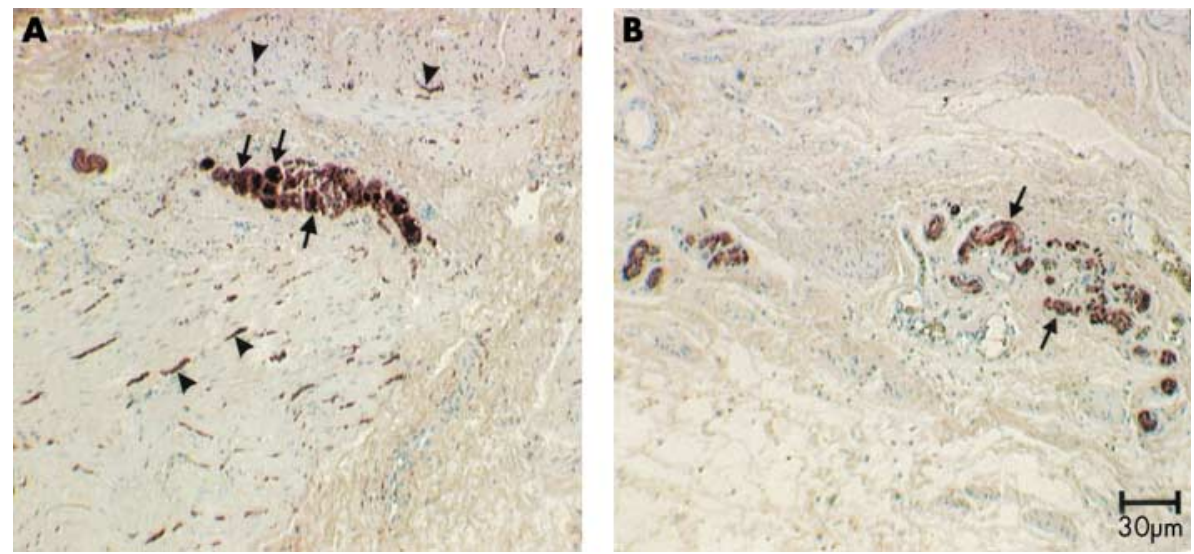

Figure 3 Protein gene product (PGP) 9.5 immunoreactivity (IR) in the corpus of a control (A) and the patient's (B) stomach. The total amount of nerve structures in (A) is greater than that in (B). Arrows show individual enteric neurones in the myenteric ganglion of control tissue. Arrowheads show PGP 9.5-IR nerve fibres in both circular and longitudinal muscle layers. Compared with control tissue, (B) shows a much weaker reaction in gastroparesis tissue. No nerve cell bodies are found in the myenteric ganglia (arrows). Very little reaction is displayed in muscle layers.

showed an increased number of lymphocytes in muscle (data not shown). Gomori's trichrome staining showed some fibrosis at the submucosae, within some ganglia, and in the muscle coat of the antrum. However, the submucous and myenteric plexus were markedly abnormal. Ganglion cells were reduced in number and size. Myenteric plexus neurones were deformed and their nuclei appeared small. PGP 9.5 immunostaining confirmed this and also showed a marked reduction in nerve fibres within the muscle layers. C-kit staining showed very few ICC, both in the myenteric plexuses and within the circular and longitudinal muscle layers of the corpus, antrum, and pylorus (figs 2,3).

Quantification analysis showed that the area of immunopositive cells for both kit and PGP 9.5 was significantly decreased in the corpus and antrum of the gastroparesis patient compared with control tissues. However, neither kit nor PGP 9.5 immunoreactivities were significantly different between the corpus and antrum (table 1).

\section{DISCUSSION}

Gastroparesis is a condition characterised by delayed gastric emptying of ingested food without gastric outlet obstruction. Many causes have been recognised as potentially interfering with normal gastric motility, with diabetes mellitus, previous gastric surgery, and IG accounting for most of these cases. Apart from a subgroup of patients reporting a viral infection
Table 1 Quantification of kit and protein gene product (PGP) immunoreactivities as a percentage of the total area

\begin{tabular}{clll}
\hline & Control & Patient & p Value \\
\hline Kit+ cells & & & \\
Corpus & $1.73(0.34)$ & $0.25(0.15)$ & $<2 \times 10^{-6}$ \\
Antrum & $1.90(0.41)$ & $0.33(0.25)$ & $<1 \times 10^{-5}$ \\
PGP+ cells & & & \\
Corpus & $4.76(1.52)$ & $1.37(0.71)$ & $<0.0005$ \\
Antrum & $4.86(1.71)$ & $1.63(0.98)$ & $<0.005$ \\
\hline
\end{tabular}

prior to the onset of symptoms, ${ }^{12}$ little is known of the pathogenesis of gastroparesis.

References to pathological studies of gastric specimens from patients with IG are almost non-existent in the medical literature. You and colleagues ${ }^{13}$ described a 26 year old woman with chronic intractable nausea and vomiting due to IG who required hemigastrectomy. Histological study of the resected specimen failed to show any discernible lesion in smooth muscle or the myenteric plexus. Pathological studies performed by Shellito and colleagues ${ }^{14}$ in four patients with severe gastroparesis also revealed no abnormalities. Therefore, 
degeneration of both enteric nerves and ICC found in our patient may provide considerable insights into this condition.

Two distinct classes of ICC in the stomach of humans and animal models are recognised. One type is scattered throughout the circular and longitudinal muscle layers from the fundus to the antrum (ICC-IM) and seems to play a key role in neurotransmission of cholinergic excitation and nitrergic inhibition. ${ }^{7}$ The second type of ICC is associated with the myenteric plexus (ICC-MY), between the longitudinal and circular muscle layers of the corpus and antrum, and is essential for slow wave generation. ${ }^{6}$ In our patient, the average overall ICC density decreased from $1.82 \%$ to $0.29 \%$ of the total area (84\% decrease). Loss of ICC-IM and associated nerves in the fundus could explain the low basal gastric tone and increased compliance. This observation is in agreement with the significant increase in gastric compliance found by Ward and colleagues ${ }^{15}$ in $\mathrm{W} / \mathrm{W}^{\mathrm{V}}$ mutant mice, which lack ICC-IM. The propagation characteristics of the peristaltic contraction of the smooth muscle cells in the antrum depend on the slow waves generated by the ICC-MY. Therefore, the antral hypomotility observed during manometry could be due to the diminished number of ICC-MY observed in the gastric specimen. ICC-IM in the pylorus were also decreased in number (data not shown). This could be caused by the same unknown mechanism affecting the rest of the stomach, although we cannot rule out a role for previous intrapyloric injection of BT. The relative contribution of ICC and enteric nerves in pyloric motor activity is not yet known. However, it is likely that a reduction in the number of ICC will impair pyloric motor activity.

Degeneration of both enteric nerves and ICC is often found in some gastrointestinal tract conditions characterised by ineffective motility of the affected segment. In a type l diabetic patient, the overall decrease in ICC density ranged from $60 \%$ to $100 \%$ depending on the area investigated. ${ }^{16}$ ICC reduction was also accompanied by loss of PGP 9.5 positive fibres. Loss of ICC was also observed in the stomach of spontaneously diabetic NOD/LtJ mice. ${ }^{17}$ In colonic biopsies from six patients with slow transit constipation, the volume of ICC in the muscle layer was reduced to $2 \%$ (normal $~ 6 \%$ ), and to $8 \%$ (normal 21\%) in the myenteric plexus region. ${ }^{18}$ ICC were also markedly reduced in one patient with colonic hypoganglionosis. ${ }^{19}$ From the present study, and the cited literature, it is clear that loss of neural structures as well as ICC can account for motor abnormalities. It should be noted that ICC and nerves develop independently, ${ }^{20-22}$ and that loss of one does not automatically result in loss of the other. Recently, the case of a patient with total aganglionosis and normal distribution of ICC has been reported ${ }^{23}$ Hence it is likely that a common factor (immune related?) caused injury to both neural and ICC networks.

From a clinical point of view, when a patient presents with symptoms consistent with gastroparesis, the diagnostic and therapeutic approach should be systematic. Upper gastrointestinal endoscopy and barium contrast studies should be obtained to rule out mechanical conditions responsible for gastric stasis. Objective assessment of gastric emptying rate should also be made. Several tests have been developed with this objective, with scintigraphy being the most accurate and convenient. Gastric motor function can be evaluated with tests that measure contractile forces (for example, antroduodenal manometry and barostat) and myoelectrical activity (for example, electrogastrography).

Therapeutic management of gastroparesis symptoms remains challenging. In mild cases, symptoms can be controlled by the combination of dietary measures and prokinetics. Recently, electrical stimulation of enteric nerves and/or pacing of slow wave activity have resurfaced. ${ }^{24}$ In some cases, pacemakers were implanted and remarkable success achieved with reduction in nausea and vomiting. However, it must be kept in mind that pacing the stomach might not improve emptying when gastroparesis is caused by insufficient excitation provided by enteric nerves.

Our patient was managed for some years with oral prokinetics but lack of control of her symptoms made intravenous erythromycin administration necessary. Erythromycin stimulates gastric myoelectrical activity in patients with gastroparesis, thereby improving gastric emptying. ${ }^{25}$ Intravenous erythromycin should be reserved for the most severe cases. Oral erythromycin is less potent and its use is limited by its antibiotic related side effects and loss of efficacy over time. ${ }^{26}$ Endoscopic injection of BT is a simple method of obtaining muscle relaxation and decreasing pyloric resistance. Following this principle, successful intrasphincteric injection of BT for severe postsurgical gastroparesis and idiopathic gastroparesis have been reported previously in the literature. ${ }^{27} 28$ In our case, this approach failed to improve either patient symptoms or gastric emptying. In rare and extreme cases, surgical palliation seems an inevitable next step. Nevertheless, surgery should be considered with extreme caution, and small bowel indemnity (both intestinal motor function and tolerance to a nutrient load) must be investigated prior to surgery.

In summary, loss of ICC and the enteric nervous system in the stomach can be involved in severe IG. In our case, degeneration of both ICC-MY and ICC-IM could explain the abnormal fundic compliance and antral hypomotility. As in many other conditions, loss of both ICC and the enteric nervous system seems to be intertwined and it is unclear whether deficiency of the former is a primary or secondary change.

\section{Authors' affiliations}

N Zárate, F Mearin, J-R Malagelada, Digestive System Research Unit, Hospital General Vall d'Hebron, Barcelona, Spain X-Y Wang, B Hewlett, J D Huizinga, , Intestinal Disease Research Program, McMaster University, Hamilton, Canada

Correspondence to: Dr F Mearin, Institute of Functional and Motor Digestive Disorders, Centro Médico Teknon, Vilana 12, 08022 Barcelona, Spain; fmearinm@meditex.es

Accepted for publication 17 December 2002

\section{REFERENCES}

1 Huizinga JD. Neural injury, repair, and adaptation in the GI tract. IV. Pathophysiology of gastrointestinal motility related to interstitial cells of Cajal. Am J Physiol 1998;275:G381-6.

2 Parkman HP, Harris AD, Krevsky B, et al. Gastroduodenal motility and dysmotility: an update on techniques available for evaluation. $\mathrm{Am} J$ Gastroenterol 1995;90:869-92.

3 Vanderwinden JM, Rumessen JJ, Liu H, et al. Intertitial cells of Cajal in human colon and in Hirschsprung's disease. Gastroenterology 1996.111:901-10.

4 Vanderwinden JM, Liu H, De Laet MH, et al. Study of the interstitial cells of Cajal in infantile hypertrophic pyloric stenosis. Gastroenterology 1996; $111: 279-88$

5 Isozaki K, Hirota S, Miyagawa J, et al. Deficiency of c-kit cells in patients with a myopathic form of chronic idiopathic intestinal pseudo-obstruction. Am J Gastroenterol 1997:92:332-4.

6 Thomsen L, Robinson TL, Lee JCF, et al. Interstitial cells of Cajal generate a rhythmic pacemaker current. Nat Med 1998;4:848-51.

7 Burns AJ, Lomax AE, Torihashi S, et al. Interstitial cells of Cajal mediate inhibitory neurotransmission in the stomach. Proc Natl Acad Sci U S A 1996;93:1208-13

8 Azpiroz F, Malagelada JR. Physiologic variations in canine gastric tone measured by an electronic barostat. Am J Physiol 1985:248:G229-37.

9 Azpiroz F, Malagelada JR. Gastric tone measured by an electronic barostat in health and postsurgical gastroparesis. Gastroenterology 1987;92:934-43

10 Malagelada JR, Stanghellini V. Manometric evaluation of functional upper gut symptoms. Gastroenterology 1985:88:1223-31.

11 Arndorfer RC, Steff JJ, Dodds WJ, et al. Improved infusion system for intraluminal esophageal manometry. Gastroenterology 1977;73:23-7.

12 Bityutskiy L, Soykan I, McCallum RW. Viral gastroparesis: a subgroup of idiopathic gastroparesis. Clinical characteristics and long-term outcomes. Am J Gastroenterol 1997;92:1501-4.

13 You CH, Chey WY, Lee KY, et al. Gastric and small intestinal myoelectric dysrhythmia associated with chronic intractable nausea and vomiting. Ann Intern Med 1981:95:449-51.

14 Shellito PC, Warshaw AL. Idiopathic intermittent gastroparesis and its surgical alleviation. Am J Surg 1984;148:408-12 
15 Ward SM, Beckett EAH, Wang XY, et al. Interstitial cells of Cajal mediate cholinergic neurotransmission from enteric motor neurons. J Neurosci 2000;20:1393-403.

$16 \mathrm{He} \mathrm{CL}$, Softer EE, Ferris CD, et al. Loss of interstitial cells of Cajal and inhibitory innervation in insulin-dependent diabetes. Gastroenterology $2001 ; 121: 427-34$

17 Ordog T, Takayama I, Cheung WK, et al. Remodeling of networks of interstitial cells of Cajal in a murine model of diabetic gastroparesis. Diabetes 2000:49:1731-9.

$18 \mathrm{He} C \mathrm{~L}$, Burgart L, Wang L, et al. Decreased interstitial cell of Cajal volume in patients with slow-transit constipation. Gastroenterology 2000;1 18:14-21

19 Faussone-Pellegrini MS, Fociani P, Buffa R, et al. Loss of interstitial cells and a fibromuscular layer on the luminal side of the colonic circular muscle presenting as megacolon in an adult patient. Gut 1999:45:775-9

20 Lecoin L, Gabella G, Le Douarin N. Origin of the c-kit positive interstitial cells in the avian bowel. Development 1996;122:725-33.

21 Young HM, Ciampoli D, Southwell BR, et al. Origin of interstitial cells of Cajal in the mouse intestine. Dev Biol 1996:96:97-107.
22 Klüppel M, Huizinga JD, Malysz J, et al. Developmental origin and Kit-dependent development of the interstitial cells of Cajal in the mammalian small intestine. Dev Dyn 1998;211:60-71.

23 Huizinga JD, Berezin I, Sircar K, et al. Development of interstitial cells of Cajal in a full-term infant without an enteric nervous system. Gastroenterology 2001;120:561-7.

24 Tougas G, Huizinga JD. Gastric pacing as a treatment for intractable gastroparesis: Shocking news? Gastroenteroly 1998;114:598-601.

25 Chen JDZ, Lin Z, Edmunds III MC, et al. Effects of octreotide and erythromycin on gastric myoelectrical and motor activities in patients with gastroparesis. Dig Dis Sci 1998;43:80-9.

26 Catnach SM, Fairclough PD. Erythromycin and the gut. Gut 1992:33:397-401.

27 Wiesel PH, Schneider R, Dorta G, et al. Botulinum toxin for refractory postoperative pyloric spasm. Endoscopy 1997;29:132.

28 Miller LS, Szych GA, Kantor SB, et al. Treatment of idiopathic gastroparesis with injection of botulinum toxin into the pyloric sphincter muscle. Am J Gastroenterol 2002;97: 1653-60.

\section{Readers' favourite}

\section{Top 10}

Click on the "Top 10" button on the homepage to see which are the best read articles each month

www.gutjnl.com 